\title{
Plasmonic Filters Based on Lithographically Patterned and Hexagonally Arranged Triangular Silver Nanoparticles Array
}

\author{
Jing Liu, ${ }^{1,2}$ Haoyuan Cai, ${ }^{1}$ Kai Tang, ${ }^{1}$ and Lingqi Kong ${ }^{2}$ \\ ${ }^{1}$ School of Information Engineering, Jimei University, Xiamen, Fujian 361021, China \\ ${ }^{2}$ Department of Physics, Xiamen University, Xiamen, Fujian 361005, China \\ Correspondence should be addressed to Jing Liu; jingliu@jmu.edu.cn
}

Received 28 September 2014; Accepted 13 November 2014

Academic Editor: Rui Vilar

Copyright (c) 2015 Jing Liu et al. This is an open access article distributed under the Creative Commons Attribution License, which permits unrestricted use, distribution, and reproduction in any medium, provided the original work is properly cited.

\begin{abstract}
A plasmonic filter applied in visible regime is proposed on the basis of hexagonally arranged triangular silver nanoparticle arrays. A method using discrete dipole approximation (DDA) to aid design parameters of the silver nanoparticle arrays is adopted for the filter design and optimization on the basis of computational numerical calculation. The influence of the particle's thickness and period on the extinction spectrum is studied using the DDA-based computational analysis. Considering the nanofabrication condition, arrays with $40 \mathrm{~nm}$ thickness and $230 \mathrm{~nm}$ period are selected and fabricated by using nanosphere lithography (NSL) technique. The experimental spectrum is basically in agreement with the theoretical spectrum derived by the DDA calculation.
\end{abstract}

\section{Introduction}

Plasmonic filters provide the unique capability of manipulating light at subwavelength scales by using versatile nanostructures, such as cubes $[1,2]$, clusters [3], shells [4], disks $[5,6]$, holes $[7,8]$, rings [9], rods [10-14], and particles with arbitrary shapes [15-17]. So far, many different types of plasmonic devices have been demonstrated, including modulators [18], interferometers [19], switches [20], polarizers [21], and absorbers [22, 23].

Plasmonic structures being used as optical filter have the following advantages [24]: the passing bandwidth is tunable through modulation of geometrical parameters and materials of the nanoparticles. This filter can work in aqueous environments such as organic chemical and biological solutions because there is no chemical reaction between the organic solution and metallic particles. This plasmonic filter is costeffective by virtue of replication technique for device fabrication. The dimensional (2D) metallic nanoparticle arrays can also be used as a high efficiency polarization filter in the visible regime by virtue of their anisotropic response to the EM wave. Wu et al. report a metamaterial-based plasmonic filter which is applicable in terahertz [25]. The realization of the plasmonic structure in terahertz ranges may lead to some applications such as a high pass filter in the terahertz imaging systems [26]. Recent advances in experimental techniques have allowed for the fabrication of nanoparticle arrays in different shapes, patterns, and sizes [27, 28]. Many research groups can fabricate different dimensional shapes of the sphere, ellipse, stick, triangle, and prism using different fabricated methods [29].

Both the thickness and the period of the metallic nanoarrays are of particular importance for surface plasmon resonances and couplings, hence affecting photon flow accordingly. $\mathrm{Fu}$ et al. proposed a rhombic Ag nanoparticles arraybased plasmonic filter for visible wavelength regime [24]. However, our study found that, compared with the rhombic Ag nanoarrays, hexagonally arranged triangular nanoarrays present more stable structure and optical properties, therefore being more suitable for plasmonic filters. What is more, the refractive index sensitivity (RIS) and figure of merit (FOM) strongly depend on the arrangement, the thickness, and period of the nanoarrays. We have done a further study on these two important factors. In this work, a new plasmonic filter working in visible wavelength regime is presented on the basis of hexagonally arranged triangular silver nanoparticle arrays with suitable thickness and period. DDA method is used to calculate the RIS and FOM of the nanostructure 
arrays with different thicknesses and periods. We develop an extended nanosphere lithography (NSL) method to fabricate the silver nanoparticle arrays with different thicknesses and periods. Our results therefore show great potential for applications in the visible wavelength regime.

\section{DDA Method}

The DDA provides a convenient method for describing light scattering from nanoparticles of arbitrary shape. In DDA formalism, the object of interest, usually called "target," is described as a cubic array lattice of electric dipoles $(N$ point dipoles) in which the polarizability and position vector of each dipole are specified as $\alpha_{i}$ and $\mathbf{r}_{i}$, respectively. The induced dipole polarization $\mathbf{P}_{i}$ in each element is determined from [30-32]

$$
\mathbf{P}_{i}=\alpha_{i} \mathbf{E}_{\mathrm{loc}, i}\left(\mathbf{r}_{i}\right), \quad i=1,2, \ldots, N,
$$

where the local field $\mathbf{E}_{\text {loc }, i}\left(\mathbf{r}_{i}\right)$ is the sum of the field radiated from all the other $N-1$ dipoles. For a given wavelength $\lambda$, including the contribution of all the other dipoles, the local field can be written as

$$
\mathbf{E}_{\mathrm{loc}, i}\left(\mathbf{r}_{i}\right)=\mathbf{E}_{0} \exp \left(i \mathbf{k} \cdot \mathbf{r}_{i}\right)-\sum_{\substack{j=1 \\ i \neq j}}^{N} \mathbf{A}_{i j} \mathbf{P}_{j}, \quad i=1,2, \ldots, N,
$$

where $\mathbf{k}$ and $\mathbf{E}_{0}$ are the wave vector and the amplitude of the incident radiation, respectively. The contribution to the electric field at position $i$ due to the dipole at position $j$ is contained in the second term on the right side of (2) and is normally expressed in terms of the dipole-dipole interaction matrix $\mathbf{A}$ as

$$
\begin{aligned}
& \mathbf{A}_{i j} \mathbf{P}_{j} \\
& =\frac{\exp \left(i \mathbf{k} r_{i j}\right)}{r_{i j}^{3}} \\
& \quad \times\left\{\mathbf{k}^{2} \mathbf{r}_{i j} \times\left(\mathbf{r}_{i j} \times \mathbf{P}_{j}\right)+\frac{1-i \mathbf{k} r_{i j}}{r_{i j}^{2}} \times\left[r_{i j}^{2} \mathbf{P}_{j}-3 \mathbf{r}_{i j}\left(\mathbf{r}_{i j} \cdot \mathbf{P}_{j}\right)\right]\right\} \\
& i=1,2, \ldots, N, \quad j=1,2, \ldots, N, \quad j \neq i,
\end{aligned}
$$

where $\mathbf{r}_{i j}$ and $r_{i j}$ correspond to the dipole-dipole position difference vector and magnitude that are defined as $\mathbf{r}_{i}-\mathbf{r}_{j}$ and $\left|\mathbf{r}_{i}-\mathbf{r}_{j}\right|$, respectively. Substituting (2) and (3) into (1), we can generate the system of equations

$$
\mathbf{A}^{\prime} \cdot \mathbf{P}=\mathbf{E}
$$

where the off-diagonal elements of the matrix $A_{i j}^{\prime}$ are the same as $A_{i j}$ and the diagonal elements of the matrix $A_{i j}^{\prime}$ are $\alpha^{-1}$.

For a system with total dipoles, $N, \mathbf{E}$ and $\mathbf{P}$ are $3 N$ dimensional vectors and $\mathbf{A}^{\prime}$ is a $3 N \times 3 N$ matrix. Solving this set of $3 N$ complex linear equations, the polarizations $\mathbf{P}_{i}$ are determined, and from this we can calculate cross section of the extinction as

$$
C_{\mathrm{ext}}=\frac{4 \pi \mathbf{k}}{\left|\mathbf{E}_{0}\right|^{2}} \sum_{i=1}^{N} \operatorname{Im}\left(\mathbf{E}_{\mathrm{loc}, i}^{*} \cdot \mathbf{P}_{i}\right) .
$$

\section{Filter Design}

In the DDA algorithm, we model the hexagonally arranged triangular silver nanoparticle arrays. This work highlights the problems in recent experiments, the geometrical parameters of the hexagonally arranged triangular silver nanoparticle arrays which cannot be decided by the experiment facture only. We will show that the calculation results are in quantitative agreement with those of the experiments. The computational calculation-based design method for determining the parameters of the hexagonally arranged triangular silver nanoparticle arrays from the fabrication point of view is provided.

Figures 1(a)-1(b) are schematic diagrams of the plasmonic filter. The DDA simulation-based design method for parameters determination of the silver particles is illustrated, as shown in Figure 1(c). It can be seen that the silver nanostructures have an in-plane width of $115 \mathrm{~nm}$ and an out-of-plane height of $40 \mathrm{~nm}$. The period of the silver nanostructure arrays is $230 \mathrm{~nm}$. These silver nanostructures lie in $y-z$ plane and the direction of the incident light is in $x$-axis. The polarization of the incident light is in $y$-axis.

In our computational calculation, we consider the silver particles with the same period and in-plane widths but different out-of-plane heights which can be fabricated by depositing metal in different thicknesses through the masks after the NSL process. The calculations are carried out for the particles arranged in a triangular structure. However, the fabricated particles may have a varied thickness/height from the design value due to fabrication error. Hence it is necessary to analyze the influence of varied thicknesses on optical performance firstly. To study this point, we calculate the extinction spectra, RIS as well as FOM of the triangular Ag nanostructure arrays with different Ag layer thicknesses. The thicknesses of triangular array structure are 30, 35, 40, and $45 \mathrm{~nm}$ with fixed period of $230 \mathrm{~nm}$. According to the calculated extinction spectra presented in Figure 2, when the Ag layer thickness increases, the intensity of the extinction and the full width at half-maximum (FWHM) decrease obviously. The positions of the peak wavelengths locate at wavelength of $691 \mathrm{~nm}$, whether the thickness is $35 \mathrm{~nm}, 40 \mathrm{~nm}$, or $45 \mathrm{~nm}$.

In order to investigate the effect of the Ag layer thickness on the sensitivity of the nanostructure array, we calculate the extinction spectra of the effective refractive index of the medium surrounding the nanostructure array. The RIS is defined as $m=\Delta \lambda / \Delta n$ [33], where $\Delta \lambda$ and $\Delta n$ denote the peak of the wavelength change and the refractive index change, respectively. For the nanostructure array with $30 \mathrm{~nm}$ thickness of Ag layer, the peak wavelength has a red shift when the refractive index $n$ increases as shown in Figure 3(a). For example, when the refractive index increases from 1.0 to 1.05 , the peak wavelength shifts from $704 \mathrm{~nm}$ to $730 \mathrm{~nm}$, exhibiting 


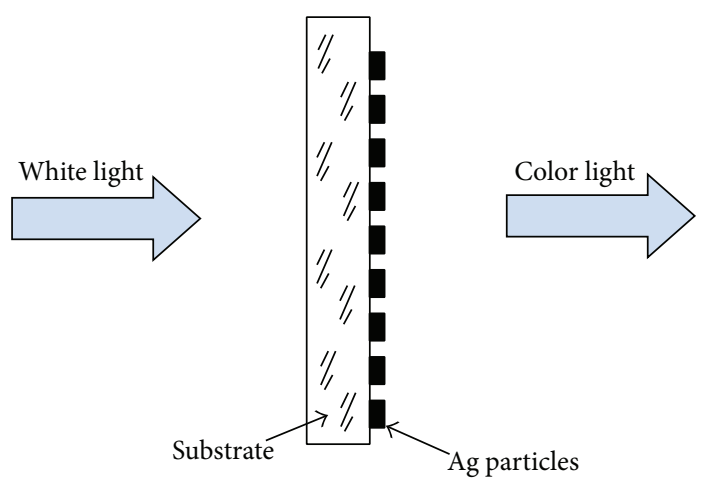

(a)

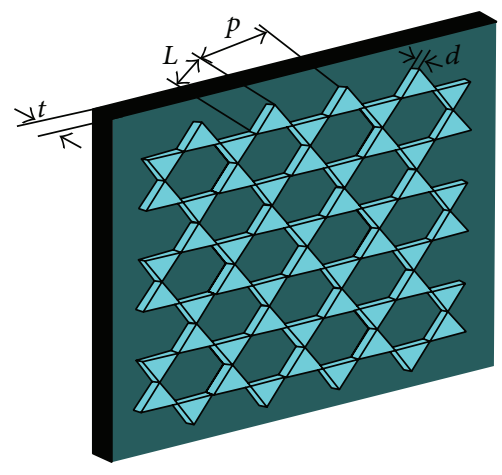

(b)

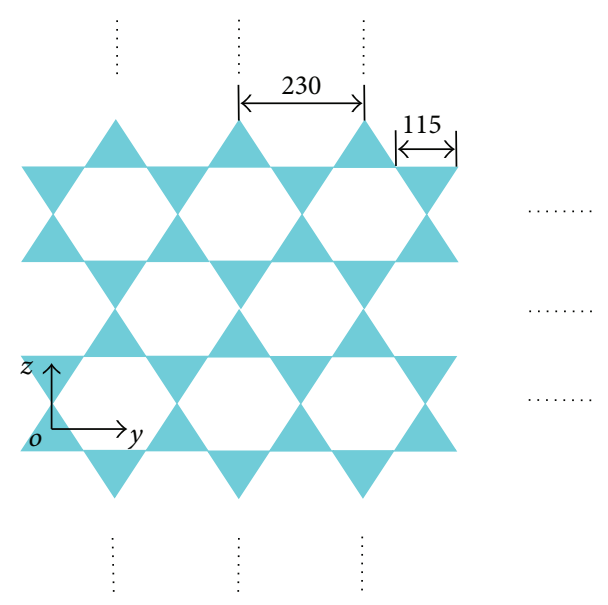

(c)

FIGURE 1: Schematic diagram of the plasmonic filter: (a) side view of the filter, (b) three-dimensional (3D) view of the filter, and (c) parameters of the DDA simulation.

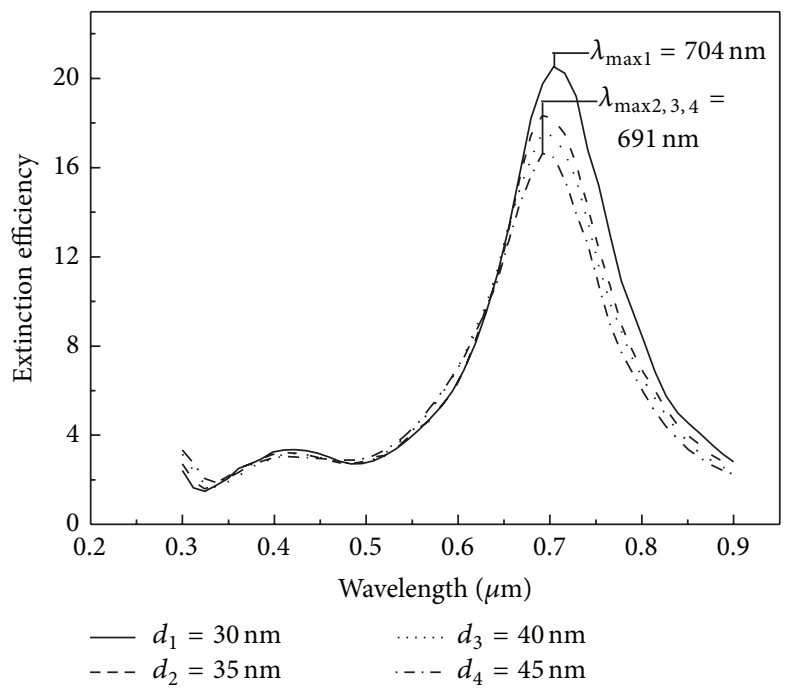

(a)

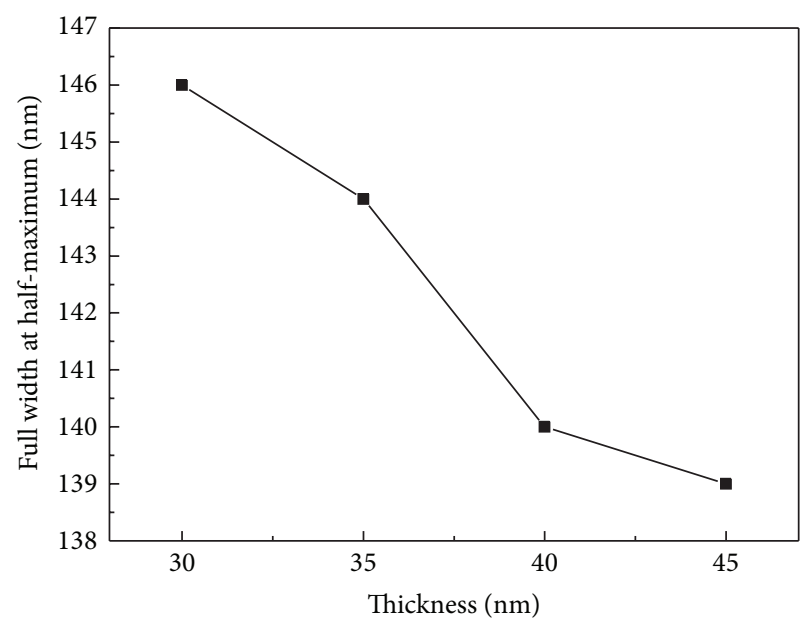

(b)

FIGURE 2: (a) Extinction spectra of triangular Ag nanoparticle arrays for different thicknesses with fixed period of $230 \mathrm{~nm}$. (b) Relationship between the peaks of wavelength and the thicknesses of triangular Ag nanoparticles. 


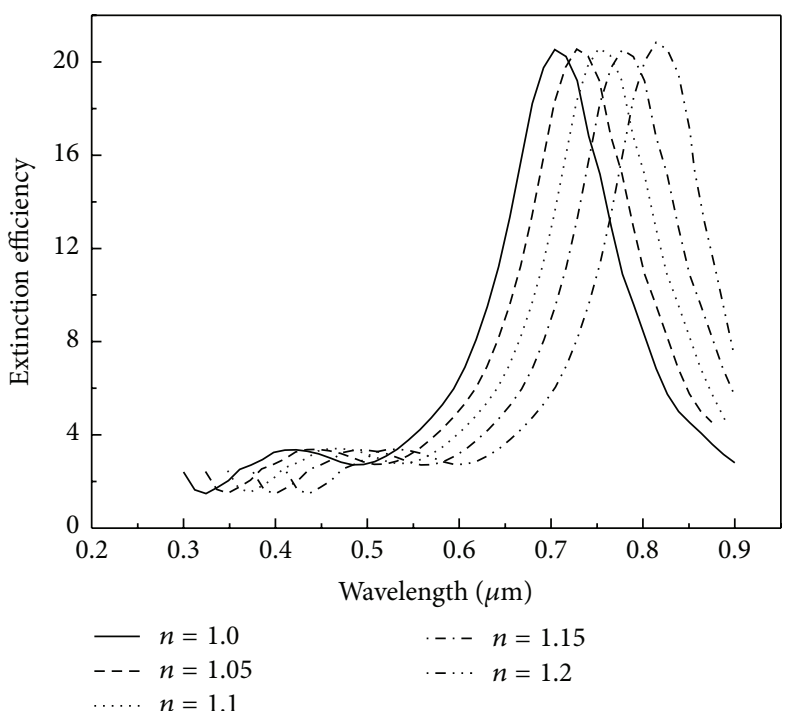

(a)

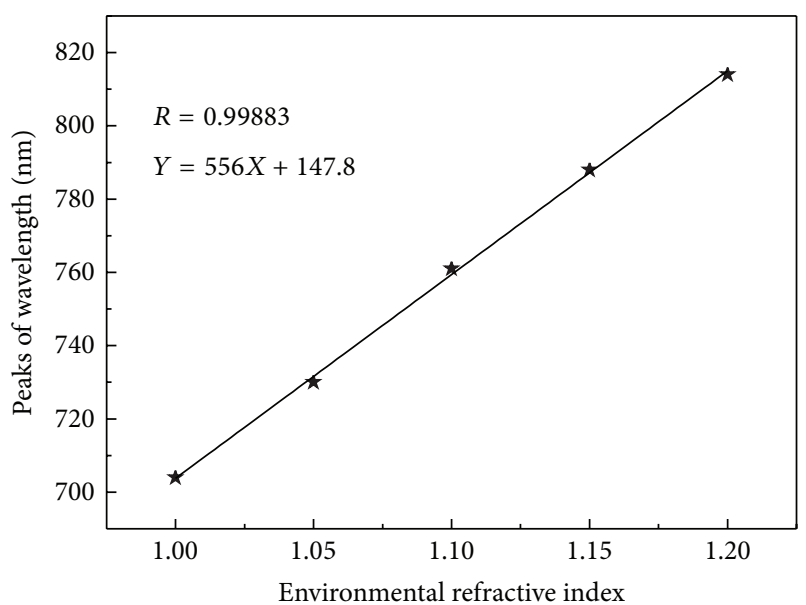

(b)

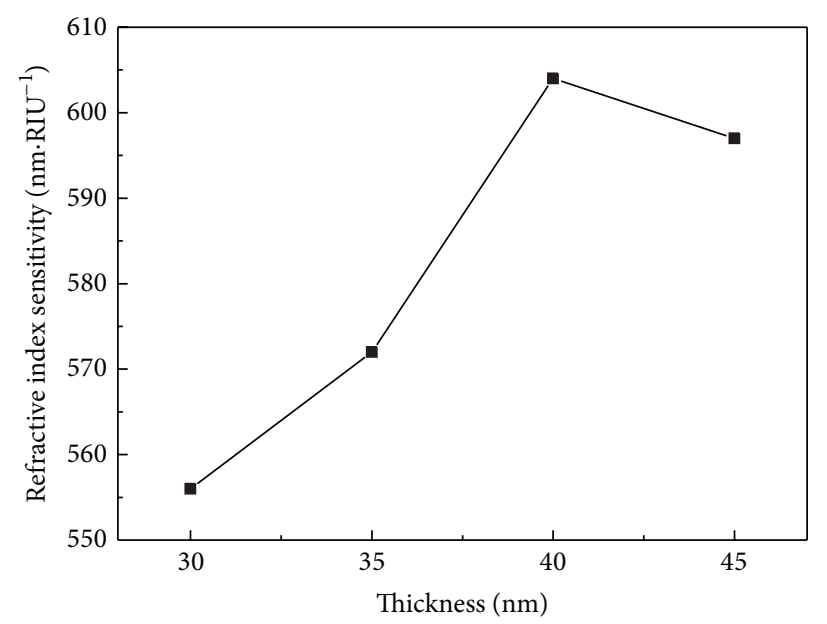

(c)

FIgURE 3: (a) Extinction spectra in different media for $30 \mathrm{~nm}$ Ag layer thickness. (b) Refractive index sensitivity curve for $30 \mathrm{~nm}$ Ag layer thickness. (c) Refractive index sensitivity distributions for different Ag layer thicknesses.

a refractive index sensitivity of $556 \mathrm{~nm} / \mathrm{RIU}$ (refractive index unit), as indicated in Figure 3(b). Figure 3(c) shows the RIS of the nanostructure arrays with different Ag layer thicknesses. The result shows that the RIS increases as the thickness of Ag layer increases from 30 to $40 \mathrm{~nm}$ and then decreases with further increase of $\mathrm{Ag}$ layer thickness to $45 \mathrm{~nm}$. When the $\mathrm{Ag}$ layer thickness is $40 \mathrm{~nm}$, the structure is very sensitive for the refractive index from 1.0 to 1.2 .

The FOM for a metal nanostructure is defined as $p=S / W$ [34], where $S$ and $W$ denote the RIS and FWHM, respectively. According to Figures 2(b) and 3(c), we calculate the FOM and the calculation result is shown in Figure 4. It can be seen from Figure 4 that the FOM of the nanostructure array remains at a more stable and relatively high value, when the thickness of Ag layer increases from 40 to $45 \mathrm{~nm}$.

In our calculation, the diverse diameters of the polystyrene spheres (PS) being used in fabrication are considered to adjust the period of the triangular silver particles. The correct direction for the facture of the hexagonally arranged triangular silver nanoparticle arrays is offered by means of analyzing variation of the particle periods. The period effects of the extinction spectra with fixed thickness of silver, $40 \mathrm{~nm}$, are calculated by changing the periods to be $230 \mathrm{~nm}$, $280 \mathrm{~nm}, 380 \mathrm{~nm}$, and $460 \mathrm{~nm}$, respectively. Figure 5 shows the resulting spectra, which look very similar to each other. The relationship between the FWHM and the period of the triangular silver particles is shown in Figure 5(b). When the periods are $230 \mathrm{~nm}, 280 \mathrm{~nm}$, and $380 \mathrm{~nm}$, the positions of the peak wavelengths are located in $691 \mathrm{~nm}$. Figure 6 shows the RIS of the nanostructure arrays with different periods. Figure 7 shows the FOM of the nanostructure arrays with different periods. As can be seen from Figure 5 to Figure 7, when the period of the nanostructure array is changed to $230 \mathrm{~nm}$, the extinction efficiency has a relatively higher value 


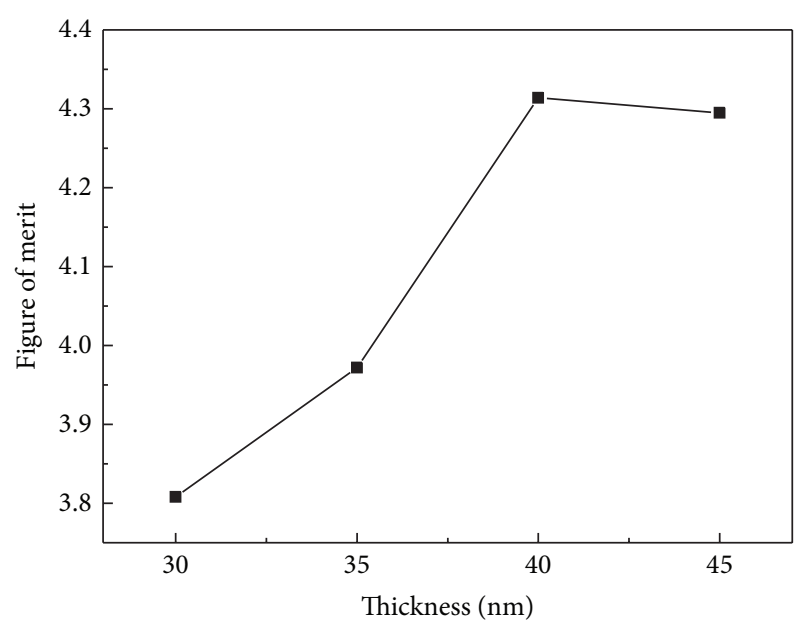

FIGURE 4: Figure of merit of the triangular Ag nanoparticle arrays with different Ag layer thicknesses.

and the corresponding FWHM of the spectrum is the widest in the periods of $280 \mathrm{~nm}, 380 \mathrm{~nm}$, and $460 \mathrm{~nm}$. We can find that the nanostructure array has maximum RIS and FOM, when the period is increased from $230 \mathrm{~nm}$ to $280 \mathrm{~nm}$. From the calculated results, we can find that the $40 \mathrm{~nm}$ thickness with $230 \mathrm{~nm}$ period is the suitable structure parameter for our calculation.

\section{Filter Fabrication}

Considering capability of the nanostructure fabrication in experiment, the thickness is selected as $30-45 \mathrm{~nm}$ and the period is selected as $230-460 \mathrm{~nm}$, respectively, in the DDA calculation.

In our experiments, the triangular metal nanostructures with different thicknesses and periods are fabricated by means of NSL technique. The PS with a mean diameter of $230 \mathrm{~nm}, 280 \mathrm{~nm}, 380 \mathrm{~nm}$, and $460 \mathrm{~nm}$ and a concentration of $10 \mathrm{wt} \%$ in solution are purchased from Suzhou NanoMicro Bio-Tech Co. Ltd. First of all, close-packed nanosphere is a prerequisite. The regular monolayer as a deposition mask is principal to achieve large-area hexagonal structure. To begin with, the PS solution is diluted to be $3 \mathrm{wt} \%$ with deionized water. The glass substrates are thoroughly cleaned, in toluene, acetone, and ethanol for $10 \mathrm{~min}$, respectively, then in piranha solution $\left(\mathrm{H}_{2} \mathrm{SO}_{4}: \mathrm{H}_{2} \mathrm{O}_{2}=3: 1\right)$ for 2 hours to remove organic residues. To achieve a hydrophilic surface, the glass substrates are ultrasonically bathed in $\mathrm{NH}_{4} \mathrm{OH}, \mathrm{H}_{2} \mathrm{O}_{2}$, and $\mathrm{H}_{2} \mathrm{O}$ solution with the ratio of $1: 1: 5$ for 2 hours. Every sonication followed rinsing with large amount of deionized water. The cleaned substrates are stored in deionized water until being used.

A single-layer of size-monodisperse PS nanospheres was drop-coated onto the substrate followed by the deposition of a silver film, as shown in Figure 8.

The deposition of metallic layer $(3 \mathrm{~N} \mathrm{Ag})$ is performed in a home-built thermal evaporator at a pressure of $5.0 \times$ $10^{-4} \mathrm{~Pa}$. The substrates are rotated at speeds of $16.5 \mathrm{rpm}$ all through the deposition. To achieve homogeneous deposition, the power for heating up of the source materials is carefully increased. The deposition rate is $\sim 2.5 \mathrm{~nm} / \mathrm{s}$ for silver layer. The thickness had been monitored using a Dektak 3 Series surface profiler to achieve an identical depth for a low reflectance. It is controlled to be $30,35,40$, and $45 \mathrm{~nm}$ for silver films, respectively. The PS spheres are lifted off by immersing in absolute ethanol for about $5 \mathrm{~s}$. The PS spheres are removed by sonication (B3500S-MT, Branson, $140 \mathrm{~W}, 42 \mathrm{kHZ}$ ) in absolute ethanol. Nanostructures of the achieved PS mask and the silver nanoparticle arrays are characterized by LEO-1530 SEM. Ultraviolet-visible (UV-vis) mirror reflection spectra are obtained on a Varian Cary 5000 UV-Vis-NIR spectrophotometer.

\section{Results and Discussion}

Figure 9 shows the SEM images of silver nanoparticle arrays for different thicknesses with fixed period of $230 \mathrm{~nm}$. As shown in Figure 9(a), the silver nanoparticles exhibit a hexagonally arranged disc structure rather than triangular structure, as the thickness of deposited silver film is $30 \mathrm{~nm}$. When the silver particles arrive at the glass substrate after passing through the triangular gaps, the energy falls sharply. In this case, the disc structure is easier formed because of its smaller surface energy. Besides, as the prior deposition particle has been combined with the substrate and no subsequent particle proceeds, the smaller disc structure cannot spread out into the triangular one. However, as the deposited film thickness increases, the angular structure mounts and tends to regular, which is presented as in Figures 9(b) and 9(c). As shown in Figure 9(c), the angular structure turns into regular, and its tip becomes sharp. This structure is very conducive to further modification experiments for signal detection. Figure 9(d) shows the SEM image of silver nanoparticle arrays with the silver film of $45 \mathrm{~nm}$ thickness. In this case, the triangular array is evident. However, the triangular tips are not as sharp as those shown in Figure 9(c), and the array is not so regular as well. This is mainly because of the deposition time, which determines the thickness of the film. Firstly, the evaporation silver particles cover the gap interspaces among the spheres, and the energy reduces greatly. Secondly, the subsequent particles combine with the advanced ones to form larger particles. Thirdly, when more and more particles accumulate, they may collapse in the triangular gaps. As their energy in the second process has decreased, the collapsed particles cannot combine with the substrate furthermore. So the particles cannot spread out into the regular triangular array, which is shown in Figure 9(d).

Different-sized silver nanoparticle arrays are presented, as shown in Figures 10(a)-10(d). The silver film thickness is fixed on $40 \mathrm{~nm}$. The nanoparticles exhibit a triangular structure and are arranged as a hexagonal array on the scale of several micrometers in lateral size. When the period is $230 \mathrm{~nm}$, the nanoparticles exhibit a triangular structure but that is more uniform than the other cases, the angular structure turns into regular, and its tip becomes sharp, as shown in Figure 10(a). This structure is very conducive to further modification experiments for signal detection. 


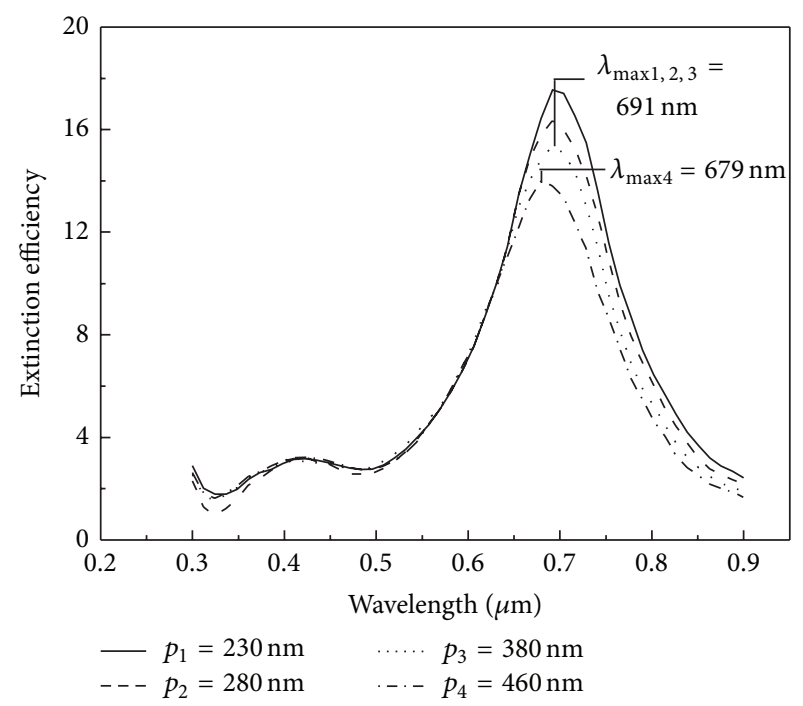

(a)

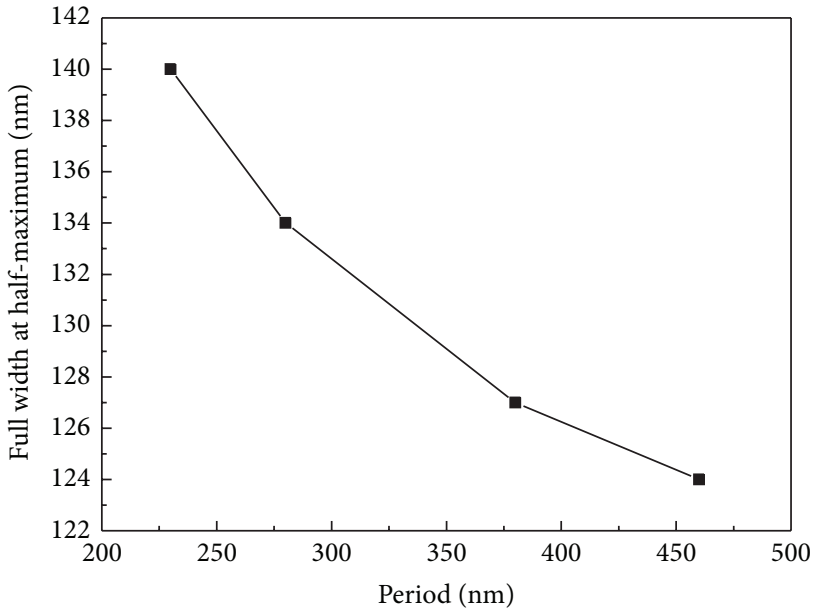

(b)

Figure 5: (a) Extinction spectra of triangular Ag nanoparticle arrays for different periods with fixed thickness of $40 \mathrm{~nm}$. (b) Relationship between the peaks of wavelength and the periods of triangular Ag nanoparticles.

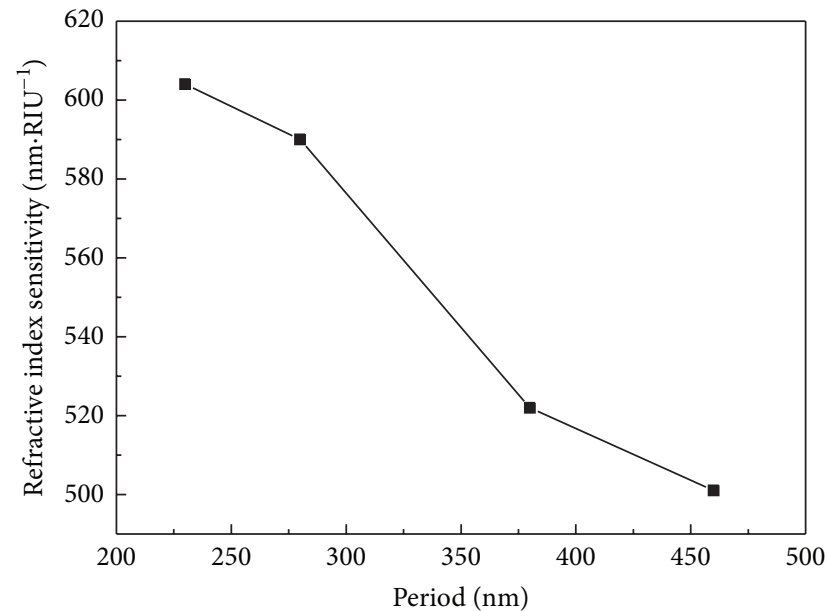

FIGURE 6: Refractive index sensitivity of the triangular Ag nanoparticle arrays with different periods.

Table 1 shows that the actual height $h_{2}$ of the nanoparticle varies with the thickness of the deposition film. As shown in Table 1, the thicknesses $h_{1}$ of the silver film of $30 \mathrm{~nm}, 35 \mathrm{~nm}$, $40 \mathrm{~nm}$, and $45 \mathrm{~nm}$ are experimental parameter according to the thermal evaporation conditions, while the actual heights $h_{2}$ of silver nanoparticles are gotten by the sample measurement. It is clear that as the thickness of the silver film is altered from $30 \mathrm{~nm}$ to $45 \mathrm{~nm}$, the actual thicknesses of the silver nanoparticle are $35 \mathrm{~nm}, 37 \mathrm{~nm}, 38 \mathrm{~nm}$, and $40 \mathrm{~nm}$, respectively. It indicates that as the silver film thickness $h_{1}$ increases, the actual height $h_{2}$ increases gradually as well. Furthermore, the nanoparticle height increases sharply when the deposited film is thin and then tends to grow slowly as the evaporation proceeds.

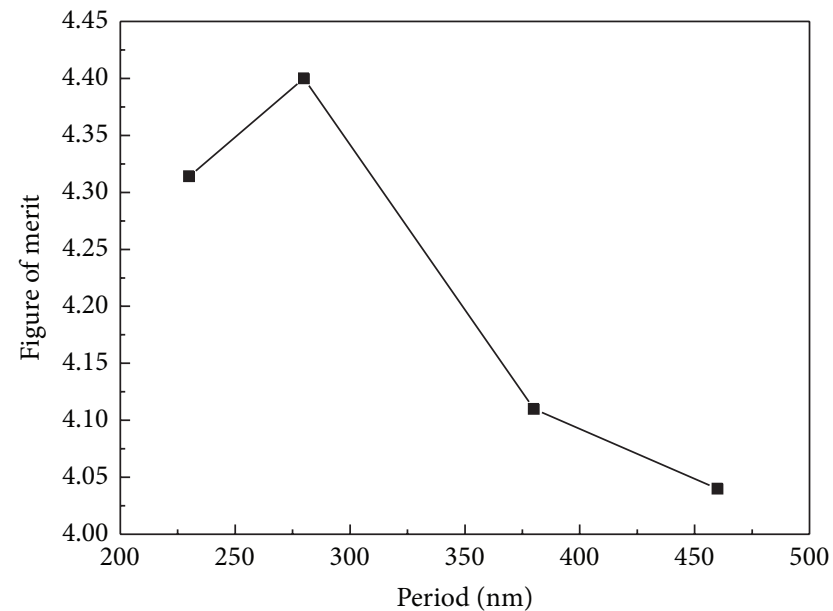

FIGURE 7: Figure of merit of the triangular Ag nanoparticle arrays with different periods.

Sub.

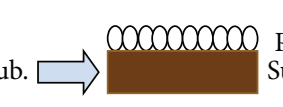

(1)

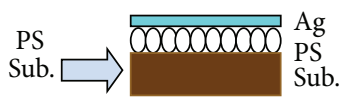

(2)
FIGURE 8: Schematic illustration of silver nanoparticle array fabrication with the following two steps: (1) drop-coating of monolayered PS nanospheres on substrate; (2) deposition of a layer of silver film over the as-coated monolayered PS nanospheres.

In our experiments, we detected the extinction efficiency of the representative triangular silver nanoparticle array with $230 \mathrm{~nm}$ period and $40 \mathrm{~nm}$ thickness of the silver particles. The experimental and the calculated results are shown in Figure 7. It can be seen apparently that when the wavelength is $663 \mathrm{~nm}$, the extinction efficiency reaches to a maximum 


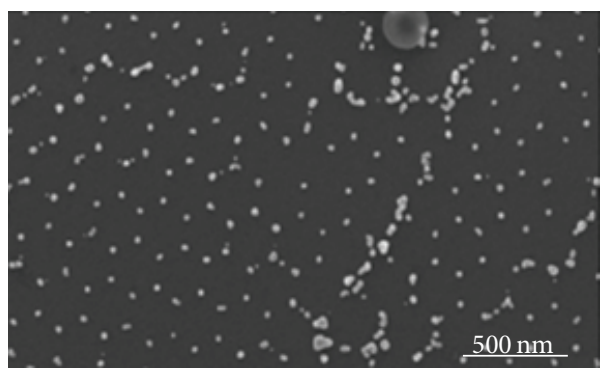

(a)

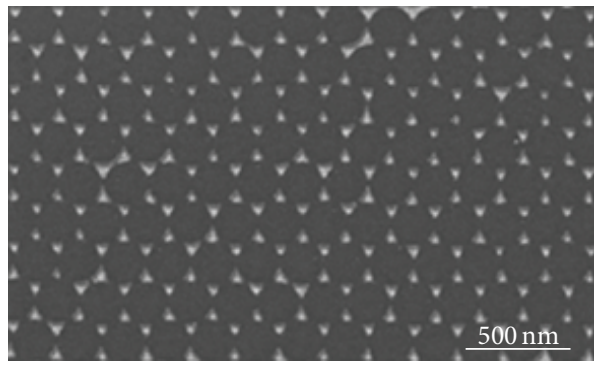

(c)

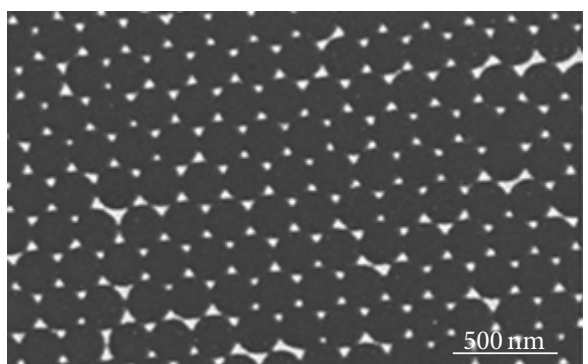

(b)

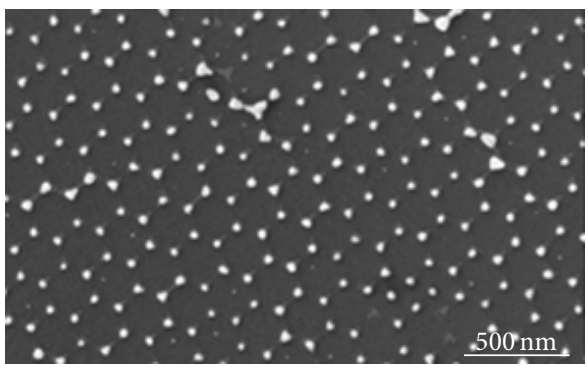

(d)

FIGURE 9: The SEM images of silver nanoparticle arrays for different thicknesses with fixed period of $230 \mathrm{~nm}$ : (a) $30 \mathrm{~nm}$; (b) $35 \mathrm{~nm}$; (c) $40 \mathrm{~nm}$, and (d) $45 \mathrm{~nm}$.

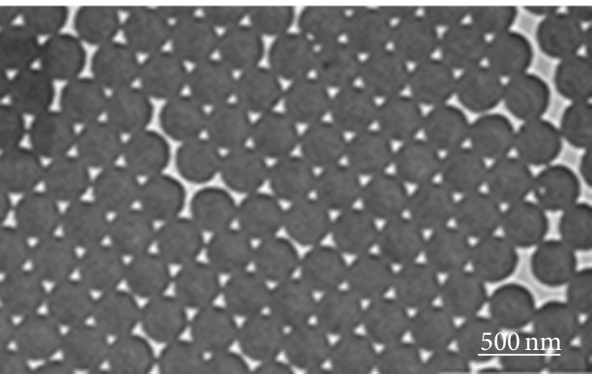

(a)

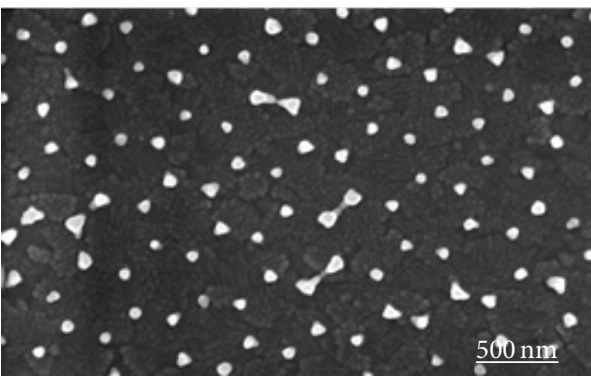

(c)

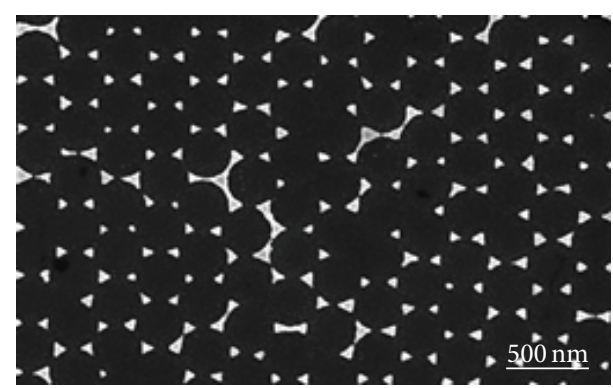

(b)

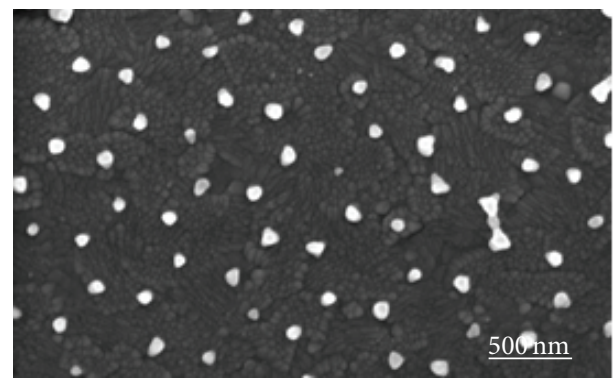

(d)

FIGURE 10: SEM images of the silver nanoarrays for different PS sphere sizes with fixed silver film thickness of $40 \mathrm{~nm}$ : (a) $230 \mathrm{~nm}$, (b) $280 \mathrm{~nm}$, (c) $380 \mathrm{~nm}$, and (d) $460 \mathrm{~nm}$.

value (Figure 11). When DDA calculation is taken to calculate the extinction efficiency, the maximum wavelength of extinction efficiency is $691 \mathrm{~nm}$. The calculated result of the plasmon wavelength with the designed model agrees with recent experimental result. The only major difference with the experiment is that the experimental peak is shifted by $28 \mathrm{~nm}$ to the blue compared to the calculated result. This is likely caused by substrate effect (see [35]). The other reason [24] is that fabrication error caused uniformity issue for both size and shape of the particles. In addition, in DDA calculated model, the edge of the triangular nanoparticles is straight while in experiment it is curving. 
TABLE 1: The actual height $h_{2}$ of the measured sample and the calculated thickness $h_{1}$ of silver film.

\begin{tabular}{lccc}
\hline Series & $\begin{array}{c}\text { The calculated } \\
\text { thickness of silver } \\
\text { film } h_{1}(\mathrm{~nm})\end{array}$ & $\begin{array}{c}\text { The actual height } \\
\text { of the measured } \\
\text { sample } h_{2}(\mathrm{~nm})\end{array}$ & $h_{2} / h_{1}$ \\
\hline $\mathrm{a}$ & 30 & 35 & 1.17 \\
$\mathrm{~b}$ & 35 & 37 & 1.06 \\
$\mathrm{c}$ & 40 & 38 & 0.95 \\
$\mathrm{~d}$ & 45 & 40 & 0.89 \\
\hline
\end{tabular}

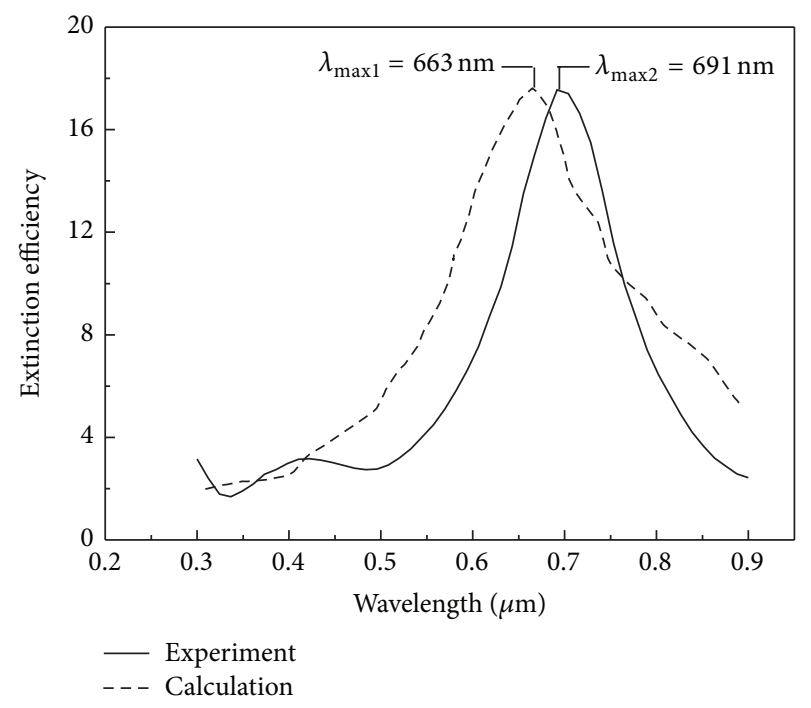

FIGURE 11: Extinction spectra of experiment and calculation.

Most of the results presented here are analysis and characterization of the thickness variation and array period. The results for the $2 \mathrm{D}$ hexagonally arranged triangular silver nanoparticle arrays show that there are suitable parameters for our experimental fabrication which are thickness of $40 \mathrm{~nm}$ and the period of $230 \mathrm{~nm}$. The experimental results are generally in agreement with the calculated results.

\section{Summary}

A hexagonally arranged triangular silver nanoparticles arraybased plasmonic filter is proposed for visible wavelength regime. A DDA algorithm-based design method is presented. After fabrication using NSL technique, spectroscopic results show that the measured spectrum is basically in agreement with the theoretical spectrum derived by the DDA calculation. Therefore, its application in the visible wavelength regime is possible.

\section{Conflict of Interests}

The authors declare that there is no conflict of interests regarding the publication of this paper.

\section{Acknowledgments}

This work was supported by Science and Technology Plan Projects of Xiamen City under Grant no. 2013S0335/ $3502 Z 20143020$ and Science and Technology Plan Projects of Fujian Provincial Science and Technology Department under Grants nos. 11151042 and 2014H0036; Science and Technology Plan Projects of Fujian Province Support The Armed Forces under Grant No. 2015.

\section{References}

[1] H. J. Chen, Z. H. Sun, W. H. Ni et al., "Fano resonance in (gold core)-(dielectric shell) nanostructures without symmetry breaking," Small, vol. 5, pp. 2111-2119, 2009.

[2] X. Kou, Z. Sun, Z. Yang, H. Chen, and J. Wang, "Curvaturedirected assembly of gold nanocubes, nanobranches, and nanospheres," Langmuir, vol. 25, no. 3, pp. 1692-1698, 2009.

[3] J. A. Fan, C. Wu, K. Bao et al., "Self-assembled plasmonic nanoparticle clusters," Science, vol. 328, no. 5982, pp. 1135-1138, 2010.

[4] J. B. Lassiter, J. Aizpurua, L. I. Hernandez et al., "Close encounters between two nanoshells," Nano Letters, vol. 8, no. 4, pp. 1212-1218, 2008.

[5] I. Zorić, E. M. Larsson, B. Kasemo, and C. Langhammer, "Localized surface plasmons shed light on nanoscale metal hydrides," Advanced Materials, vol. 22, no. 41, pp. 4628-4633, 2010.

[6] Y. J. Liu, Y. B. Zheng, J. Liou, I.-K. Chiang, I. C. Khoo, and T. J. Huang, "All-optical modulation of localized surface plasmon coupling in a hybrid system composed of photoswitchable gratings and au nanodisk arrays," The Journal of Physical Chemistry C, vol. 115, no. 15, pp. 7717-7722, 2011.

[7] T. W. Ebbesen, H. J. Lezec, H. F. Ghaemi, T. Thio, and P. A. Wolff, "Extraordinary optical transmission through sub-wavelenght hole arrays," Nature, vol. 391, no. 6668, pp. 667-669, 1998.

[8] Y. J. Liu, E. S. P. Leong, B. Wang, and J. H. Teng, "Optical transmission enhancement and tuning by overylaying liquid crystals on a gold film with patterned nanoholes," Plasmonics, vol. 6, no. 4, pp. 659-664, 2011.

[9] A. R. Halpern and R. M. Corn, "Lithographically patterned electrodeposition of gold, silver, and nickel nanoring arrays with widely tunable near-infrared plasmonic resonances," ACS Nano, vol. 7, no. 2, pp. 1755-1762, 2013.

[10] H. Chen, L. Shao, Q. Li, and J. Wang, "Gold nanorods and their plasmonic properties," Chemical Society Reviews, vol. 42, no. 7, pp. 2679-2724, 2013.

[11] Y. Zheng, M. Xiao, S. Jiang, F. Ding, and J. Wang, "Coating fabrics with gold nanorods for colouring, UV-protection, and antibacterial functions," Nanoscale, vol. 5, no. 2, pp. 788-795, 2013.

[12] G. A. Wurtz, R. Pollard, W. Hendren et al., "Designed ultrafast optical nonlinearity in a plasmonic nanorod metamaterial enhanced by nonlocality," Nature Nanotechnology, vol. 6, no. 2, pp. 107-111, 2011.

[13] G. A. Wurtz, W. Dickson, D. O’Connor et al., "Guided plasmonic modes in nanorod assemblies: strong electromagnetic coupling regime," Optics Express, vol. 16, no. 10, pp. 7460-7470, 2008.

[14] D. P. Lyvers, J.-M. Moon, A. V. Kildishev, V. M. Shalaev, and A. Wei, "Gold nanorod arrays as plasmonic cavity resonators," ACS Nano, vol. 2, no. 12, pp. 2569-2576, 2008. 
[15] A. M. Kern and O. J. F. Martin, "Excitation and reemission of molecules near realistic plasmonic nanostructures," Nano Letters, vol. 11, no. 2, pp. 482-487, 2011.

[16] J. P. Kottmann, O. J. F. Martin, D. R. Smith, and S. Schultz, "Spectral response of plasmon resonant nanoparticles with a non-regular shape," Optics Express, vol. 6, no. 11, pp. 213-219, 2000.

[17] A. Lovera and O. J. F. Martin, "Plasmonic trapping with realistic dipole nanoantennas: analysis of the detection limit," Applied Physics Letters, vol. 99, no. 15, Article ID 151104, 2011.

[18] J. A. Dionne, K. Diest, L. A. Sweatlock, and H. A. Atwater, "PlasMOStor: a metal-oxide-Si field effect plasmonic modulator," Nano Letters, vol. 9, no. 2, pp. 897-902, 2009.

[19] Y. K. Gao, Z. M. Xin, Q. Q. Gan, X. H. Cheng, and F. J. Bartoli, "Plasmonic interferometers for label-free multiplexed sensing," Optics Express, vol. 21, no. 5, pp. 5859-5871, 2013.

[20] Y. J. Liu, Q. Hao, J. S. T. Smalley, J. Liou, I. C. Khoo, and T. J. Huang, "A frequency-addressed plasmonic switch based on dual-frequency liquid crystals," Applied Physics Letters, vol. 97, no. 9, Article ID 091101, 2010.

[21] Y. Zhao, M. A. Belkin, and A. Alù, "Twisted optical metamaterials for planarized ultrathin broadband circular polarizers," Nature Communications, vol. 3, pp. 870-879, 2012.

[22] K. Aydin, V. E. Ferry, R. M. Briggs, and H. A. Atwater, "Broadband polarization-independent resonant light absorption using ultrathin plasmonic super absorbers," Nature Communications, vol. 2, pp. 517-524, 2011.

[23] Y. Zhao, Q. Hao, Y. Ma et al., "Light-driven tunable dual-band plasmonic absorber using liquid-crystal-coated asymmetric nanodisk array," Applied Physics Letters, vol. 100, no. 5, pp. 053119-053126, 2012.

[24] Y. Q. Fu, S. L. Zhu, X. L. Zhou, and W. Zhao, "Rhombic silver nanoparticles array-based plasmonic filter," International Journal of Modern Physics B, vol. 25, no. 19, pp. 2557-2566, 2011.

[25] D. Wu, N. Fang, C. Sun et al., "Terahertz plasmonic high pass filter," Applied Physics Letters, vol. 83, no. 1, pp. 201-203, 2003.

[26] V. M. Lubecke, K. Mizuno, and G. M. Rebeiz, "Micromachining for terahertz applications," IEEE Transactions on Microwave Theory and Techniques, vol. 46, no. 11, pp. 1821-1831, 1998.

[27] R. F. W. Pease, "Nanolithography and its prospects as a manufacturing technology," Journal of Vacuum Science \& Technology $B$, vol. 10, pp. 278-285, 1992.

[28] J. Melngailis, A. A. Mondelli, I. L. Berry III, and R. Mohondro, "A review of ion projection lithography," Journal of Vacuum Science and Technology B: Microelectronics and Nanometer Structures, vol. 16, no. 3, pp. 927-957, 1998.

[29] J. C. Hulteen, D. A. Treichel, M. T. Smith, M. L. Duval, T. R. Jensen, and R. P. Van Duyne, "Nanosphere lithography: sizetunable silver nanoparticle and surface cluster arrays," Journal of Physical Chemistry B, vol. 103, no. 19, pp. 3854-3863, 1999.

[30] R. Jin, Y. Cao, C. A. Mirkin, K. L. Kelly, G. C. Schatz, and J. G. Zheng, "Photoinduced conversion of silver nanospheres to nanoprisms," Science, vol. 294, no. 5548, pp. 1901-1903, 2001.

[31] B. T. Draine and P. J. Flatau, "Discrete-dipole approximation for scattering calculations," Journal of the Optical Society of America A: Optics and Image Science, and Vision, vol. 11, no. 4, pp. 14911499, 1994.

[32] B. T. Draine and P. J. Flatau, User Guide for the Discrete Dipole Approximation Code DDSCAT.7.3.0, 2008.
[33] S. Zhu, C. Du, and Y. Fu, "Localized surface plasmon resonancebased hybrid Au-Ag nanoparticles for detection of Staphylococcus aureus enterotoxin B," Optical Materials, vol. 31, no. 11, pp. 1608-1613, 2009.

[34] L. J. Sherry, S.-H. Chang, G. C. Schatz, R. P. Van Duyne, B. J. Wiley, and Y. Xia, "Localized surface plasmon resonance spectroscopy of single silver nanocubes," Nano Letters, vol. 5, no. 10, pp. 2034-2038, 2005.

[35] C. Chaumet, A. Rahmani, and G. W. Brvant, "Generalization of the coupled dipole method to periodic structures," Physical Review B, vol. 67, pp. 165404-165411, 2003. 

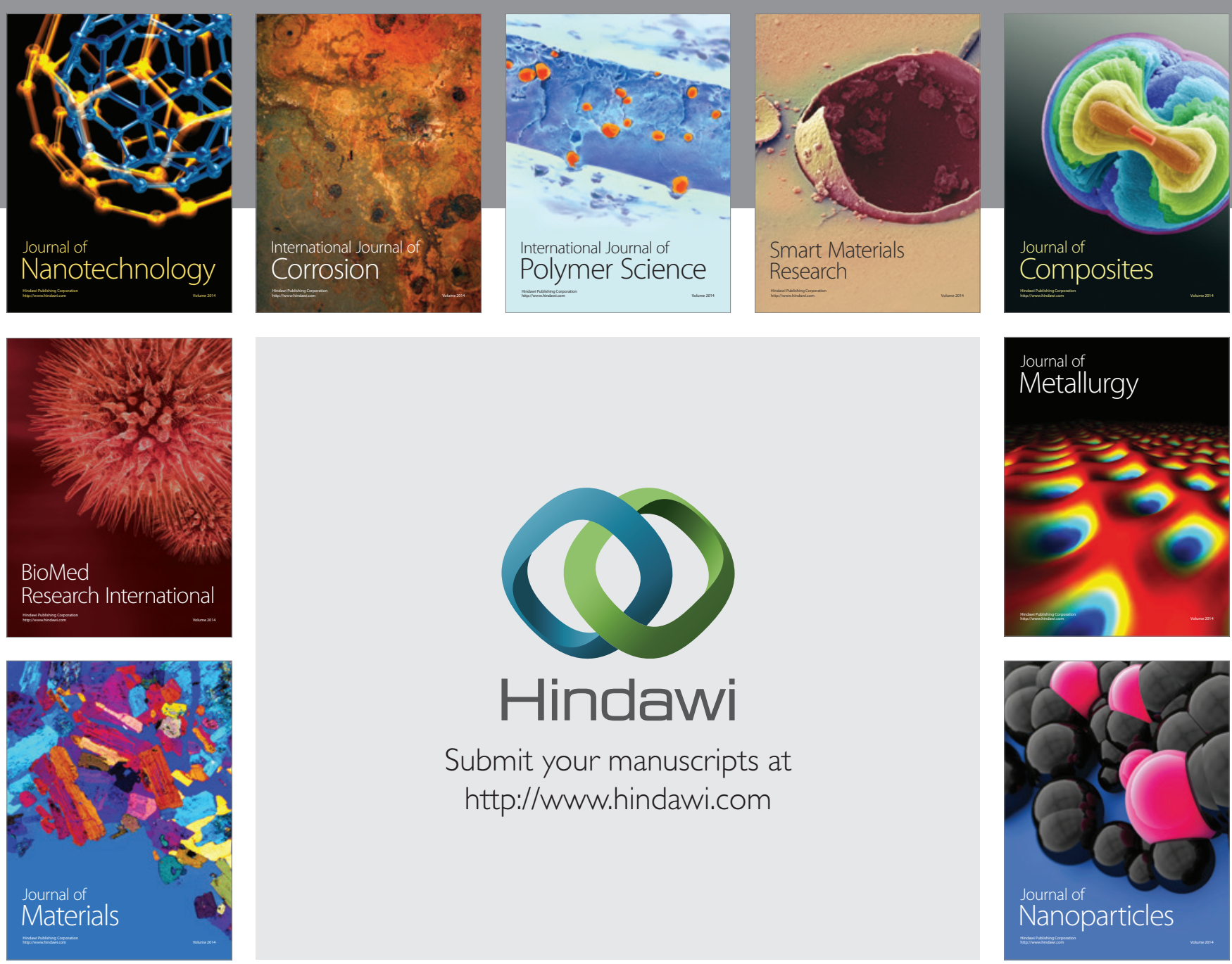

Submit your manuscripts at http://www.hindawi.com
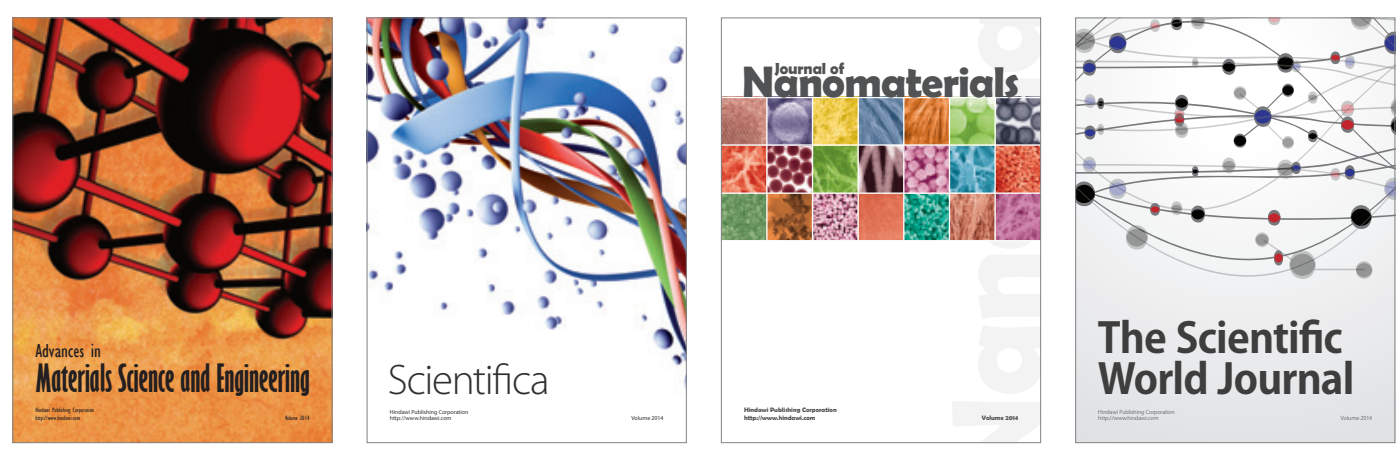

\section{The Scientific World Journal}
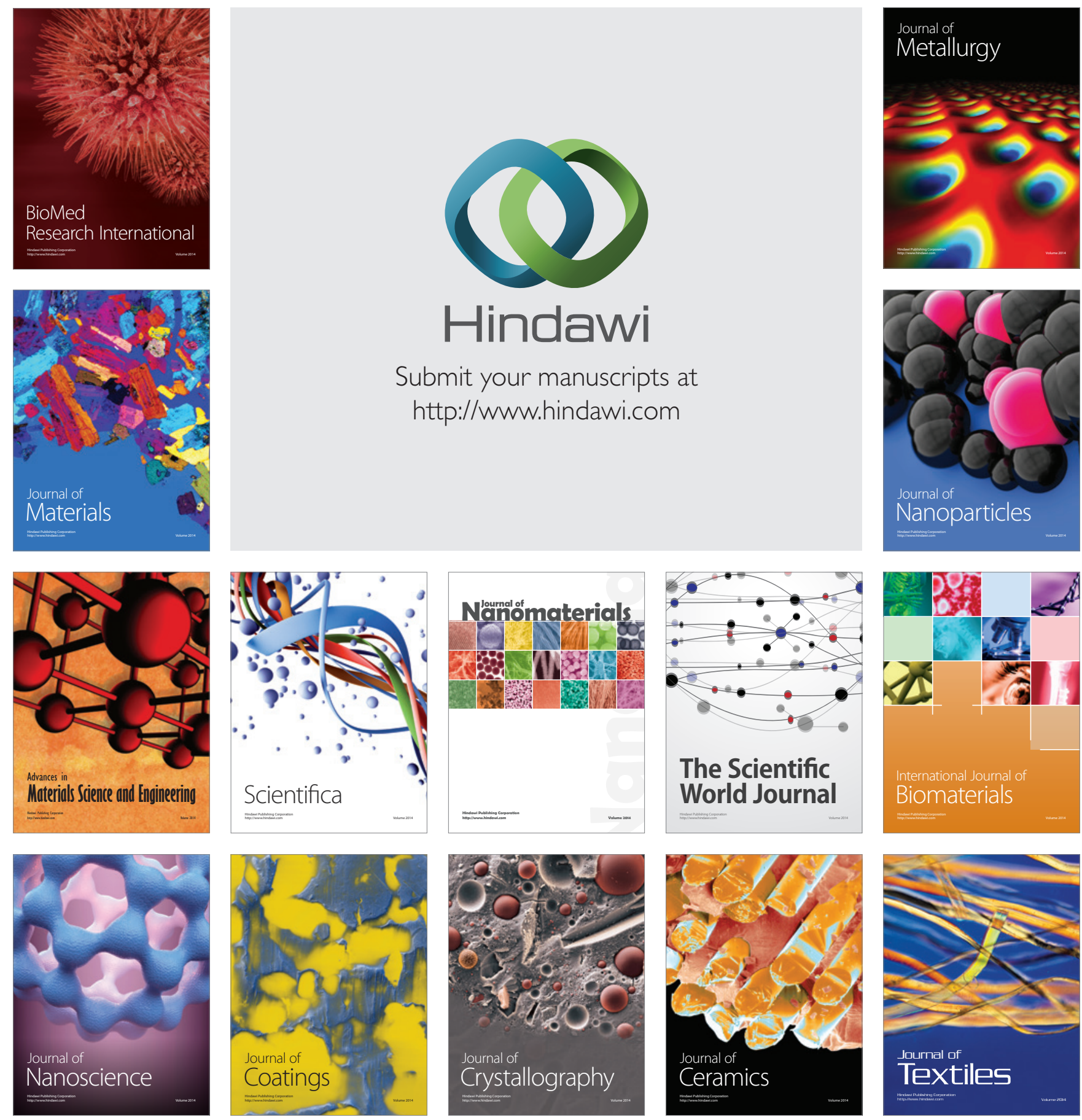